\title{
Diffraction results from the ATLAS experiment
}

\author{
Marek Taševský, on behalf of the ATLAS Collaboration \\ Institute of Physics, Academy of Sciences of the Czech republic, Na Slovance 2, 18221 Prague, \\ Czech republic
}

\begin{abstract}
First studies dealing with diffraction processes published by the ATLAS collaboration are discussed. Two analyses are reviewed, both relying on data from proton-proton collisions at $\sqrt{s}=7 \mathrm{TeV}$ from a part of data taking in 2010 where the effect of pile-up background was negligible. One analysis reports on a measurement of the rapidity gap distribution compared with diffractive and hadronization models, the second on the measurement of the total inelastic cross section.
\end{abstract}

Keywords: inelastic scattering, single diffraction, rapidity gap

PACS: $13.85 . \mathrm{Hd}$

\section{INTRODUCTION}

Although the cross section for diffractive processes forms about $25 \%$ of the total inelastic proton-proton cross section at LHC, they are still not well understood. The key point in discussing the first diffractive results at LHC is a need for an unambiguous experimental definition of diffractive signature which would be acceptable and reproducible by theorists. The two analyses discussed below show such attempts, one using single-sided events, the other rapididity gaps. Larger gaps between particles can be due to statistical fluctuations in the hadronization process, which are exponentially suppressed [1] as a function of gap size, or by t-channel colour singlet exchange (CSE). The CSE can be provided by electroweak or more frequently by strongly interacting states. The latter processes are termed as diffractive and their phenomenological description is inspired by Regge theory where the CSE is associated with the Pomeron [2,3]. Inclusive inelastic (hereafter called Minimum Bias (MB)) processes are formed by two classes, the nondiffractive (ND) and diffractive processes, where the latter is divided further according to where the gap is found, to Single dissociation (SD) (one gap close to the intact leading proton), Double dissociation (DD) (one gap in the central rapidity region) or Central diffraction (CD) (both intact leading protons accompanied by one gap). The kinematics of the diffractive dissociation is commonly described in terms of the four-momentum transfer squared, $t$, and momentum fraction loss of the intact leading proton, $\xi$, calculated as $M^{2} / s$ where $M$ is the invariant mass of the dissociated system and $s$ is the squared centre-of-mass energy. The gap between the intact proton and the dissociated system is related to $\xi$ by the relation $\Delta \eta \simeq-\ln \xi$. Prospects for the diffractive program in the ATLAS experiment were outlined e.g. in [4].

\section{RAPIDITY GAPS IN SOFT PROCESSES}

Rapidity gaps in this analysis [5] were reconstructed using the calorimeter system of the ATLAS detector [6] consisting of the electromagnetic (EM) $(|\eta|<3.2)$, hadronic (H) 
end-cap $(1.5<|\eta|<3.2)$ and EM/H forward system $(3.1<|\eta|<4.9)$, complemented by the steel/scintillator hadronic tile calorimeter $(|\eta|<1.7)$. The calorimetric information is then combined with the information from the inner tracking detector (ID) for $|\eta|<2.5$. An interval in $\eta$ is considered to be empty of final state particles if it does not contain clusters that pass noise suppression requirements for respective calorimeter subsystems and have $p_{T}>p_{T}^{\text {cut }}$ or good tracks in ID with $p_{T}>p_{T}^{\text {cut }}$. Detector simulation showed that the lowest accessible energy with a good acceptance is for clusters with $p_{T}>200 \mathrm{MeV}$. The MB events are selected using the Minimum Bias Trigger Scintillator (MBTS) consisting of two discs symetricaly placed $\pm 3.56 \mathrm{~m}$ away from the ineraction point (IP) and covering $2.1<|\eta|<3.8$. The trigger efficiency is larger than $99 \%$ for particles produced in this $\eta$ region. The forward gap size $\eta_{F}$ is defined by the larger of the two empty rapidity regions extending between the edges of the detector acceptance at $\eta=4.9$ or -4.9 and the nearest track or calorimeter cluster passing the selection requirements at smaller $|\eta|$.
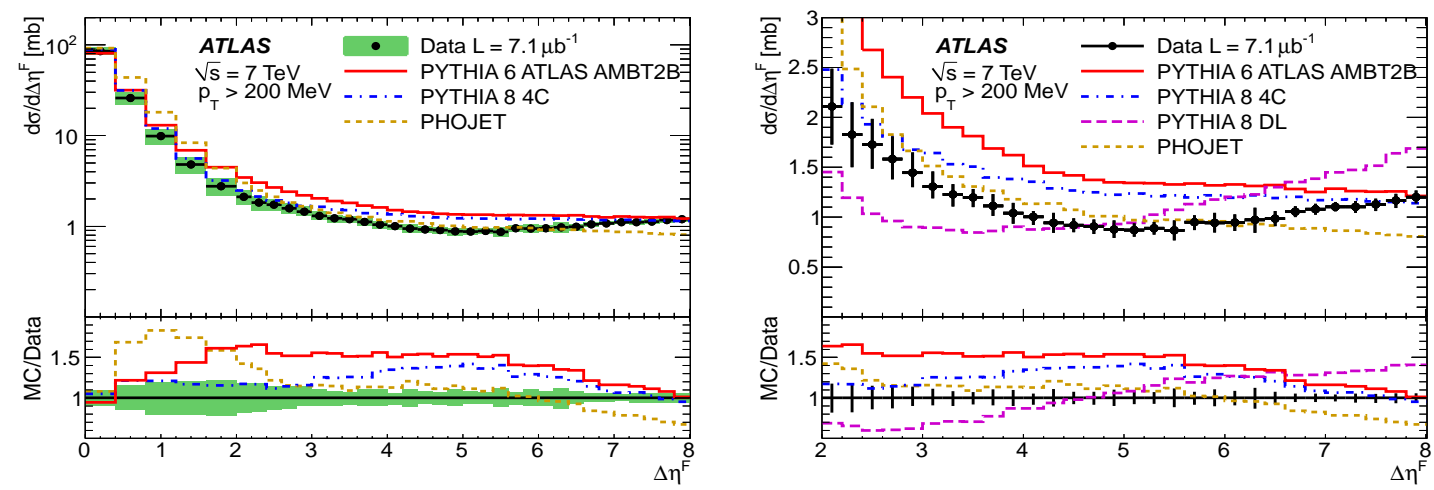

FIGURE 1. Left: Inelastic cross section as a function of the forward gap size $\Delta \eta_{F}$ for particles with $p_{T}>200 \mathrm{MeV}$ compared with predictions of PHOJET and PYTHIA 6 and 8. Right: a zoom of the left plot into the region $\Delta \eta_{F}>2$. Plots taken from [5].

Results corrected for detector effects to the level of stable particles and for $p_{T}^{\text {cut }}=200$ $\mathrm{MeV}$ are shown in Figs. 1 and 2. In Fig. 1 the measured differential inelastic cross section $d \sigma / d \Delta \eta_{F}$ is compared to predictions of PYTHIA 6 and 8 and PHOJET. The data show an exponential decrease for $\Delta \eta_{F}<2.5$ attributed to ND processes and a plateau for $\Delta \eta_{F}>3$ typical for diffractive processes. Although all models follow these general features seen in data, they all fail to describe both the shape and normalization over the whole gap range. This is not surprising since none of these models have been tuned to describe diffractive processes at LHC and this measurement can serve this purpose. The plot on the right gives information about dynamics (expressed by pomeron intercept $\alpha_{P}(0)$ ) of observed diffractive processes at large gaps, namely by studying the slope at this region. The slope is fitted in the region $\Delta \eta_{F}>5$ using PYTHIA 8 with Donnachie-Landshoff model (DL) of diffractive scattering [7]. The fitted value is $\alpha_{P}(0)=1.058 \pm 0.003$ (stat. ${ }_{-0.039}^{+0.034}$ (syst.) which due to large systematic uncertainties is still compatible with the default DL value 1.085 [7]. The slower rise of the gap distribution predicted by the other models in this region is explained by the fact that they all used the $\alpha_{P}(0)=1.0$ setting.

The distribution at small rapidity gaps is sensitive to fluctuations in hadronization 

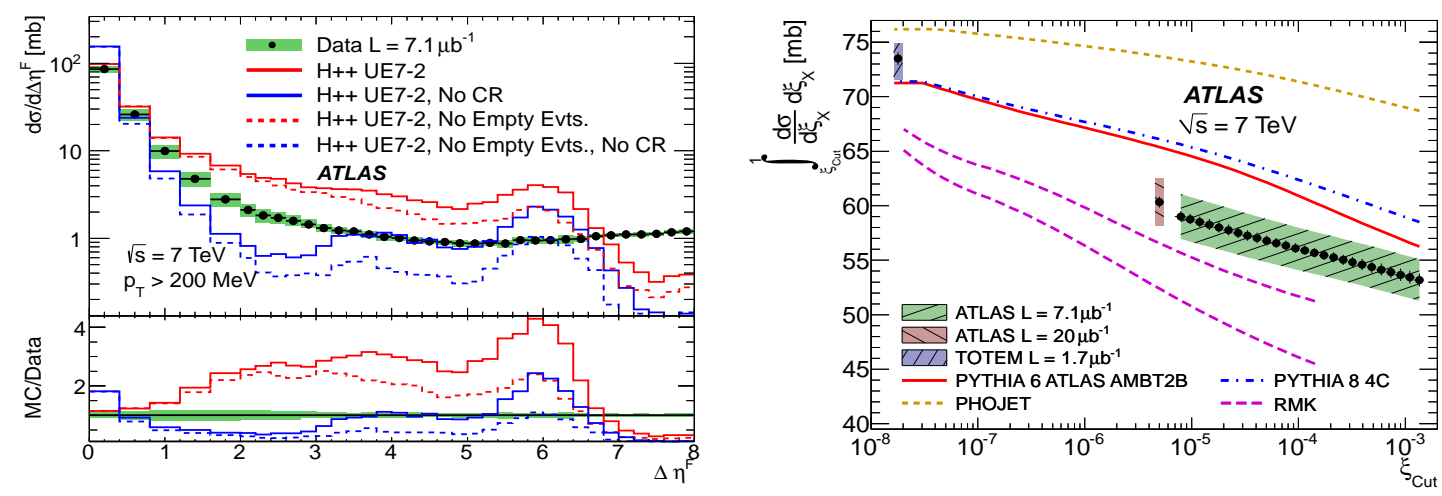

FIGURE 2. Left: Inelastic cross section as a function of the forward gap size $\Delta \eta_{F}$ for particles with $p_{T}>200 \mathrm{MeV}$ compared with predictions of HERWIG++ UE7-2 tune [5]. Right: Inelastic integrated cross section as a function of minimum $\xi$ denoted as $\xi_{\text {cut }}$.

processes thus it is interesting to compare the data to other hadronization models than the Lund String model implemented in PYTHIA and PHOJET. The predictions of HERWIG++ based on the cluster hadronization model and in the tune MB UE7-2 [5] are compared to data in Fig. 2 left. It should be emphasized that this version of HERWIG++ does not contain any diffractive processes and thus only the exponential fall is expected. Although not containing any explicit diffractive component, HERWIG++ produces large gaps with non-exponential behaviour and a bump at $\Delta \eta_{F}=6$. This behavior persists when the Colour reconnection (CR) model is switched off or events with no scatters present in the HERWIG++ underlying event model are excluded (No Empty Evts).

Thanks to the strong correlation $\Delta \eta \simeq-\ln \xi$, one can convert the inelastic cross section integrated over an interval $0<\Delta \eta_{F}<\Delta \eta_{F}^{c u t}$ into the integral over the interval $\xi_{\text {cut }}<\xi<1$. The result is shown in Fig. 2 right where after applying small hadronization corrections and including particles with $p_{T}<200 \mathrm{MeV}$ (for details see [5]), the integrated cross section is shown as a function of $\xi_{c u t}$. The data are compared to a previous ATLAS measurement [8] and the TOTEM experiment (TOTal and Elastic cross section Measurement) determination of the total inelastic cross section [9] from the measurement of the elastic cross section via the optical theorem. From a comparison to theoretical models shown in Fig. 2 we conclude that two KMR models [10], although not correct in normalization, exhibit a better agreement in shape with data than PYTHIA and PHOJET which underestimate the fraction of low mass diffractive events.

\section{SINGLE SIDED MINIMUM BIAS EVENTS}

Another possibility to enhance the diffractive signal in an event sample is to require no activity (above a noise threshold) in a part of the detector. In the analysis [8] discussed elsewhere in these proceedings [11] this is achieved by vetoing the activity in the MBTS on one side of IP and requiring at least one track in the ID with $p_{T}>0.5 \mathrm{GeV}$. The measured ratio of such single-sided to all events, $R_{S S}=(10.0 \pm 0.4) \%$, when compared to various models, requires the fraction of diffractive to all inelastic processes to lie in 
the range $25-30 \%$ depending on the model.

\section{PROSPECTS FOR PROTON TAGGING}

Further and the purest possibility to extract diffractive events is to tag the intact leading protons. Here we briefly remind of the ALFA detector [12] whose primary aim is collecting elastic events. A good sample of such data is now under processing and study. Besides that, depending on the LHC optics and if combined with the whole ATLAS detector, ALFA can collect soft as well as hard diffractive events. Processes of interest are e.g. production of identified particles such as $\Lambda$ or $K_{S}$, of dijets where one can study the underlying event, or multi-jets that provide information on multi-parton interactions. A possible upgrade project AFP for collecting diffractive and exclusive signal at high pile-up is discussed elsewhere in these proceedings [13].

\section{SUMMARY}

In this note, three methods to enhance diffractive contribution in data from inclusive $p p$ collisions at $\sqrt{s}=7 \mathrm{TeV}$ are mentioned. We concentrate on the rapidity gap method. The main conclusion is that the measured distributions of neither $\Delta \eta_{F}$ nor of $\xi$ are well described by any of the available models. This is not surprising because they have not been tuned to diffractive LHC data yet but the first analyses of diffractive processes at the LHC reported in these proceedings may certainly be used for this tuning. We remind of possible measurements of the soft and hard diffraction processes using the ALFA detector and refer also to a possible upgrade project AFP to install forward proton detectors that should operate at high pile-up environment.

\section{ACKNOWLEDGMENTS}

Supported by the projects LA08032 and LA08015 of the Ministry of Education of the Czech republic.

\section{REFERENCES}

1. J. Bjorken, S. Brodsky, H. J. Lu, Phys. Lett., B286 (1992) 153.

2. E. Feinberg, I. Pomerancuk, Suppl. Nuovo Cimento, 3 (1952) 652.

3. G. Chew, S. Frautschi, Phys. Rev. Lett., 7 (1961) 394.

4. M. Taševský, Nucl. Phys. Proc. Suppl. 179-180 (2008) 187; ATL-PHYS-CONF-2008-019.

5. ATLAS Collaboration, Eur. Phys. J. C72 (2012) 1926.

6. ATLAS Collaboration, J. Inst. 3 (2008) S08003.

7. A. Donnachie, P. Landshoff, Nucl. Phys. B244 (1984) 322.

8. ATLAS Collaboration, Nature Commun. 2 (2011) 463.

9. TOTEM Collaboration, EPL (Europhysics Letters), 96 (2011) 21002.

10. M. G. Ryskin, A. D. Martin, V. A. Khoze, Eur. Phys. J., C71 (2011) 1617.

11. T. Zenis, these proceedings.

12. ATLAS Collaboration, CERN/LHCC/2008-004, January 2008.

13. L. Chytka, these proceedings. 